\title{
BRAF mutation as a biomarker in colorectal cancer
}

This article was published in the following Dove Press journal:

Advances in Genomics and Genetics

15 October 2015

Number of times this article has been viewed

\section{Anna M Varghese \\ Leonard B Saltz \\ Department of Medicine, Division of Solid Tumor Oncology, Gastrointestinal Oncology Service, Memorial Sloan Kettering Cancer Center, New York, NY, USA}

Correspondence: Anna M Varghese Department of Medicine, Division of Solid Tumor Oncology, Gastrointestinal Oncology Service, Memorial Sloan Kettering Cancer Center, 300 East 66th Street, New York, NY I0065, USA Email varghesa@mskcc.org
Abstract: Nearly $10 \%$ of colorectal cancers (CRCs) harbor mutations in $B R A F$. While cytotoxic chemotherapy remains central to the treatment for patients with metastatic $\mathrm{CRC}$, there is a growing understanding that $\mathrm{CRC}$ is comprised of molecularly and clinically distinct populations. $B R A F$-mutant CRC is one such subset. We are learning more about the complexity of $B R A F$ mutant CRCs and the ways in which patients with $B R A F$-mutant CRCs may or may not benefit from targeted therapies. This article reviews the role of $B R A F$ as a biomarker in CRC and its implications for treatment.

Keywords: personalized medicine, colorectal cancer, $B R A F$

\section{Overview of CRC}

In 2015, an estimated 132,700 people will be diagnosed with CRC and nearly 50,000 people will die of this disease. ${ }^{4}$ Treatment for stage I, stage II, and stage III disease is curative in intent and includes surgery, adjuvant cytotoxic chemotherapy to prevent recurrent disease for a subset of patients with stage II colon cancers and nearly all patients with stage III CRCs, and radiation for many patients with stage II and stage III rectal cancers. For patients with metastatic or stage IV CRCs that are not surgically resectable, treatment is palliative and the goal is to improve disease-related symptoms and to prolong life. Treatments include systemic chemotherapy with drugs such as 5-fluorouracil, oxaliplatin, and irinotecan, as well as administration of bevacizumab and ziv-aflibercept, which are anti-vascular endothelial growth factor agents. For patients with $R A S$-wild-type $(R A S$-WT) tumors, monoclonal antibodies directed at the epidermal growth factor receptor (EGFR), cetuximab and panitumumab, can be added to systemic chemotherapy.

\section{Biology of EGFR/KRAS/BRAF signaling}

The molecular landscape in CRC is complex with genetic alterations seen in the genes encoding the RAS family of proteins (KRAS and NRAS), the RAF kinases (ARAF, BRAF, and CRAF), phosphatidylinositol-3-kinase (PI3K), EGFR, human epidermal growth factor receptor 2 (HER2), and others. The most commonly seen and readily targetable alterations center on the EGFR/RAS/RAF signaling pathway.

EGFR is a member of the ErbB family of receptor tyrosine kinases. Activation of the transmembrane receptor of EGFR results in autophosphorylation and dimerization of the receptor, activating the RAS family proteins (HRAS, KRAS, and NRAS). This in turn regulates multiple downstream pathways critical for cell survival and proliferation, including, but not limited to, the RAF family of kinases (ARAF, BRAF, and CRAF) and PI3K. The RAF kinases comprise a key regulatory pathway, which then 
activates the downstream MEK and ERK pathways. Alterations at nearly each of the kinases listed earlier can be seen in patients with $\mathrm{CRCs}$, and each represents an opportunity to target and personalize therapies. ${ }^{5-7}$

\section{Biomarkers in CRC}

The most widely known biomarkers in CRC are KRAS and $N R A S$, and $32 \%-40 \%$ and $2 \%-5 \%$ of CRCs, respectively, have alterations in these genes. The vast majority of $K R A S$ alterations are in codons 12 and 13., ${ }^{2,8}$ Activating mutations in $R A S$ cause EGFR-independent, constitutive activation of downstream pathways, resulting in uncontrolled cell survival and proliferation. Consequently, patients with $R A S$ mutant CRCs do not receive benefit from EGFR-directed therapies. ${ }^{2,8-13}$ EGFR-directed therapies, such as cetuximab and panitumumab administration, have been shown to improve response rate and overall survival only for patients with $R A S$-WT CRCs. ${ }^{2,8-13}$ Given these treatment implications of the presence or absence of $R A S$ mutations, testing for $K R A S$ and NRAS mutations is standard in the care of patients with metastatic CRCs.

\section{BRAF alterations in cancer}

Activating $B R A F$ mutations, most commonly in codon 600 , are present in many solid tumors, including CRCs $(\sim 10 \%)$, melanomas $(\sim 50 \%)$, and lung cancers $(\sim 1 \%-2 \%) .{ }^{1-3,14,15}$ Single-agent inhibition of BRAF via vemurafenib or dabrafenib has changed the way we treat patients with melanoma and lung cancer. Sosman et $\mathrm{al}^{16}$ performed a single-arm, Phase II study of patients with $B R A F$-mutant melanoma. These patients with $B R A F$-mutant melanoma received the BRAF inhibitor vemurafenib and they experienced a median overall survival of 15.9 months and response rates of $53 \% .{ }^{16}$ Chapman et al ${ }^{17}$ performed a randomized Phase III study of patients with $B R A F$-mutant melanoma, in which patients were randomized to receive either vemurafenib or the standard of care at the time, a cytotoxic chemotherapy called dacarbazine. They demonstrated that patients receiving vemurafenib had improved response rates and overall survival compared to patients receiving dacarbazine, with overall survival improving from 9.7 months to 13.6 months. ${ }^{17,18}$ On the basis of these studies, vemurafenib and dabrafenib have been approved for the treatment of patients with $B R A F$-mutant melanoma. In patients with $B R A F$-mutant lung cancers, promising case reports of dramatic clinical and radiographic responses, similar to the responses seen in patients with melanoma, have been published. ${ }^{19-21}$ Initial results from a Phase II study ${ }^{22}$ evaluating dabrafenib in patients with $B R A F$-mutant lung cancers found that $33 \%(28 / 84)$ had a partial response. Although not yet approved in $B R A F$-mutant lung cancer, dabrafenib has been given a breakthrough designation by the US Food and Drug Administration for consideration of rapid approval in the treatment of patients with $B R A F$-mutant lung cancers. While single-agent inhibition of BRAF has been promising and effective for patients with melanoma and lung cancers, treatment of $B R A F$-mutant $\mathrm{CRC}$ has been more challenging.

\section{BRAF in CRC Clinical and pathologic features of patients with BRAF-mutant CRC}

Patients with $B R A F$-mutant CRCs are characterized by unique clinical and pathologic characteristics. Although the absolute numbers in each study are small, several features characteristic of $B R A F$-mutant colon cancer have become apparent. In the largest retrospective study to date, ${ }^{23}$ mutation status, clinical outcomes, and clinicopathologic features were determined and analyzed in patients participating in the N0147 study. The N0147 study evaluated the role of various adjuvant chemotherapy regimens administered to patients after surgery for stage III (lymph node-positive) colon cancer. ${ }^{23}$ Of 2,166 colon cancers undergoing $B R A F$ testing, 310 (14\%) had $B R A F$ V600E mutations. Compared to patients with $B R A F-\mathrm{WT}$ tumors, patients with $B R A F$-mutant colon cancers were more likely to be $\geq 70$ years old ( $34 \%$ vs $14 \%, P<0.001$ ), female ( $63 \%$ vs $45 \%, P<0.001)$, white ( $94 \%$ vs $85 \%, P<0.001)$, and a current or former smoker ( $62 \%$ vs $52 \%, P<0.001)$. Patients with $B R A F$-mutant colon cancers are also more likely to have right-sided tumors ( $86 \%$ vs $48 \%, P<0.001)$. These tumors are also more likely to be characterized by a poorly differentiated or with high-grade histology (47\% vs $22 \%, P<0.001$ ), and more invasive $(14 \%$ vs $10 \%, P<0.04) .{ }^{23}$ These findings are consistent with other studies on patients with $B R A F$-mutant colon cancers and their tumors. ${ }^{1,24-26}$ In CRC, BRAF mutations have been found to be mutually exclusive with $R A S$ mutations. $^{23,27}$

Additionally, the pattern of metastatic spread at diagnosis appears unique in this patient population compared to that in patients with $B R A F$-WT tumors. Liver involvement at the time of diagnosis was less common $(60 \%$ vs $80 \%, P<0.01)$, and peritoneal involvement was more common $(26 \%$ vs $14 \%, P<0.01) .{ }^{26}$ Axillary lymph node involvement, a highly unusual site of metastatic disease for $\mathrm{CRC}$, has also been seen in patients with $B R A F$-mutant $C R C$. In a retrospective review 
of 100 patients with $B R A F$-mutant $\mathrm{CRC}$ at Memorial Sloan Kettering Cancer Center, nine patients had axillary lymph node metastasis. ${ }^{28}$

$B R A F$-mutant CRC tumors were also more likely to be deficient in DNA mismatch repair. ${ }^{23,26}$ As described by Gonsalves et al, ${ }^{23}$ in the case of 310 patients with $B R A F$-mutant CRCs, $47 \%$ of patients had DNA mismatch repair-deficient tumors, compared to $7 \%$ of patients with $B R A F$-WT tumors $(P<0.001)$. Abnormalities in the DNA mismatch repair pathway are identified by assessing for the presence of microsatellite instability or performing immunohistochemical staining for the presence or absence of proteins important in the DNA mismatch repair pathway. While mismatch repair deficiency is often associated with germline-inherited cancer predisposition syndromes, such as Lynch syndrome (hereditary nonpolyposis colorectal cancer or HNPCC), mismatch repair deficiency can also occur secondary to somatic alterations in genes important for DNA mismatch repair. Although the association among $B R A F \mathrm{~V} 600 \mathrm{E}$-mutant $\mathrm{CRC}$, mismatch repair deficiency, and tobacco history has not been completely clarified, some have argued that tobacco exposure among patients with $B R A F$-mutant CRCs results in increased DNA methylation, subsequent silencing of these methylated genes that may be important in mismatch repair, and, ultimately, development of the molecular and immunohistochemical phenotype of a DNA mismatch repair-deficient tumor. ${ }^{23}$ Consequently, if a DNA mismatch repair-deficient tumor harbors a $B R A F-\mathrm{V} 600 \mathrm{E}$ mutation, there is virtual certainty that the DNA mismatch repair status is probably secondary to a somatic alteration in the DNA rather than a germline change associated with an underlying cancer predisposition syndrome, and germline testing for Lynch syndrome is therefore not indicated.

\section{Prognostic implications of $B R A F$ mutations}

$B R A F$ mutation status is consistently associated with poor prognosis in multiple retrospective evaluations. Using data from the PETACC-3 randomized Phase III trial evaluating the utility of adding irinotecan to 5-fluorouracil/leucovorin for the adjuvant treatment of $\mathrm{CRC}$, the prognostic value of $B R A F$ mutations was evaluated..$^{29}$ Of 3,278 patients enrolled in this trial, 1,307 had $B R A F$ testing performed prospectively. Eight percent $(103 / 1,307)$ had evidence of $B R A F$ mutations. While no difference was seen between patients with $B R A F$-WT and $B R A F$-mutant colon cancers with respect to recurrence-free survival, patients with $B R A F$-mutant, DNA mismatch repair-intact CRC had poorer overall survival, with an increased hazard ratio of 2.2. ${ }^{29}$ Additionally, in a retrospective evaluation of 229 patients with metastatic CRCs undergoing treatment with systemic chemotherapy, overall survival was 11 months for patients with $B R A F$-mutant CRCs $(\mathrm{n}=15)$ compared to 41 months for patients with $K R A S$-WT/ $B R A F$-WT tumors $(\mathrm{n}=135) .{ }^{24}$ Overall survival for patients with $B R A F$-mutant CRCs is typically measured at less than 1 year. $23,26,30$

While patients with $B R A F$-mutant CRC clearly have a poorer prognosis, Popovici et $\mathrm{al}^{31}$ evaluated whether gene expression can clarify the underlying biology and clinical course of patients with both $B R A F$-mutant and $B R A F$-WT CRCs. Using a gene expression classifier incorporating 64 genes, a population of patients with $B R A F$-WT CRCs who had similar gene expression profiles as patients with $B R A F$-mutant CRCs was identified. These patients had similarly poor outcomes, suggesting an underlying and poorly understood biologic similarity between these molecularly distinct patient populations.

\section{Treatment implications of BRAF mutations}

Regarding standard chemotherapy treatment decisions, the presence of $B R A F$ mutations in CRC is thought to play no role in the sensitivity of tumors to standard cytotoxic chemotherapy, such as oxaliplatin and irinotecan. ${ }^{32}$ In the MRCFOCUS trial, which compared first-line treatments with 5-fluorouracil, 5-fluorouracil/oxaliplatin, and 5-fluorouracil/ irinotecan, patients with $B R A F$-mutant CRCs had a shorter overall survival compared to patients with $B R A F$-WT CRCs; however, no association was seen between the presence of $B R A F$ mutations and response to chemotherapy with oxaliplatin versus irinotecan. ${ }^{32}$ On the basis of these data, $B R A F$ mutation plays no role in the choice of standard, cytotoxic chemotherapy. ${ }^{32}$

Regarding response to standard, targeted therapies, growing evidence suggests that $B R A F$ mutations in CRC predict lack of response to EGFR-directed therapies, despite the fact that these CRCs are $R A S$-WT, and EGFR-directed therapies are effective for patients with $R A S$-WT CRCs, as demonstrated in the CRYSTAL trial for cetuximab and in other prospective and retrospective evaluations of the use of EGFRdirected therapies for patients with $R A S$-WT CRCs. ${ }^{12,13,25,33-35}$ There is growing evidence to suggest that $B R A F$ alterations predict lack of response or, at a minimum, lack predictive value in relation to $B R A F$ mutations and EGFR-directed 
therapies. In an evaluation of the CRYSTAL and OPUS trials evaluating the role of cetuximab in the first-line treatment of patients with CRC, BRAF mutations were identified in 70/800 patients, and no significant differences were seen in treatment outcomes for patients with $B R A F$-mutant and $B R A F$-WT CRC receiving EGFR-directed therapy. ${ }^{35}$ De Roock et al evaluated tumor samples from 773 patients with CRC treated with cetuximab, in addition to performing comprehensive genotyping of $K R A S, B R A F, N R A S$, and $P I K 3 C A{ }^{8}$ Of 36 patients found to have $B R A F$-mutant CRC, only $8 \%$ of patients responded to treatment $(2 / 24)$ with cetuximab. In other retrospective studies of patients with metastatic CRC treated with EGFR-directed therapies, patients whose tumors harbored $B R A F$ alterations did not receive benefit from EGFR-directed therapies. ${ }^{30,36,37}$ These studies, however, are all limited in their retrospective nature and overall small numbers of patients with $B R A F$-mutant $C R C$, with each study having between nine and 24 patients with $B R A F$-mutant CRC receiving EGFR-directed therapy.

To address this issue, several meta-analyses have been undertaken to further understand whether the presence or absence of $B R A F$ mutations can be linked to response to EGFR-directed therapies. One meta-analysis evaluating the role of EGFR-directed therapies in patients with $B R A F$ mutant CRCs has noted that the response rate to EGFRdirected therapies in $B R A F$-mutant patients compared to $B R A F-\mathrm{WT}$ patients was 0.14 (95\% confidence interval [CI]: 0.04-0.53), suggesting that patients with $B R A F$-mutant CRCs do not respond to EGFR-directed therapy. ${ }^{38}$ Similarly, another meta-analysis of nine Phase III trials and one Phase II trial with 463 patients with $B R A F$-mutant CRCs revealed that the addition of EGFR-directed therapies did not improve response rate, progression-free survival, or overall survival. ${ }^{39}$ These findings suggest that the presence of $R A S$ or $B R A F$ mutations in CRC predicts lack of response to EGFR-directed therapies, and we recommend that comprehensive evaluation for the presence or absence of $B R A F$ and $R A S$ alterations should be performed at the time of diagnosis of metastatic disease to determine whether patients will benefit from EGFR-directed therapies.

This recommendation is consistent with that of the National Comprehensive Cancer Network, which "strongly recommends genotyping of tumor tissue in all patients with metastatic CRC for RAS (KRAS exon 2 and non-exon 2; NRAS) and $B R A F$ at diagnosis of stage IV disease. ${ }^{40}$ Historically, testing for molecular alterations in patients with CRC was performed via molecular testing of individual genetic alterations in $K R A S$. Given the need for molecular analysis of multiple genes, including $K R A S, N R A S$, and $B R A F$, more comprehensive molecular testing is needed. ${ }^{41}$ Options can include mutationprofiling assays to identify "hotspot" mutations that are seen frequently in these and other oncogenes, using technologies such as Sequenom ${ }^{\mathrm{TM}}$ mass spectrometric genotyping and polymerase chain reaction-based assays. ${ }^{42-44}$ Another more comprehensive option is the use of next-generation sequencing technology in targeted panels of important cancer-associated genes, including $B R A F, K R A S, N R A S$, and hundreds of others, to identify mutations as well as gene amplifications, deletions, and fusions. ${ }^{45-47}$ The clinical significance and treatment implications of many of these findings remain unclear in the standard care of patients with CRC. ${ }^{41,45-47}$

Given the poor outcomes in patients with $B R A F$-mutant $\mathrm{CRCs}$, Yaeger et $\mathrm{al}^{26}$ sought to understand the role of metastasectomy in these patients. Although resection of limited metastatic disease can provide cures for patients with metastatic $\mathrm{CRCs}$, their data indicated that patients with $B R A F$ mutant CRCs had a nonsignificant shorter recurrence-free survival after metastasectomy compared to that in patients with $B R A F$-WT tumors ( 7 months compared to 11 months, $P<0.084)$ and a significantly shorter overall 2 -year survival (61\% compared to $86 \%, P=0.003) .{ }^{26}$ Similar findings are noted by Renaud et al, ${ }^{48}$ who evaluated 180 patients with metastatic CRC who underwent resection of lung metastases. Patients with $B R A F$-mutant CRCs were found to have a significantly worse median overall survival of 15 months compared to that of patients with $K R A S$-mutant and KRAS-WT/ $B R A F$-WT CRCs (55 months and 98 months, respectively). These findings raise concern that metastasectomy in patients with $B R A F$-mutant CRCs is far less likely to provide the durable responses and cures seen in some patients with $B R A F$-WT CRCs and, thus, suggest that $B R A F$ mutation may be a relative contraindication to metastastectomy with curative intent.

\section{Targeting BRAF in CRC Single-agent targeting of $B R A F$ in CRC}

Given the remarkable responses for single-agent BRAF inhibitors in patients with $B R A F$-mutant melanomas and lung cancers, evaluations of single-agent BRAF inhibitors were undertaken in CRC. Disappointingly, no meaningful clinical activity was seen in patients with $B R A F$-mutant CRCs. In a Phase I study of vemurafenib, ${ }^{49} 21$ patients with $B R A F$-mutant CRCs were treated. Although the drug was well tolerated, it was essentially inactive, with only one patient having a confirmed partial response. Median progressionfree survival was 3.7 months. Five patients did have a mixed 
response, although these findings are certainly less promising than the results of BRAF inhibition and melanoma. ${ }^{49}$ These findings suggest that $B R A F$-mutant $\mathrm{CRC}$ is more complex than those other cancers in which single-agent targeting of BRAF is sufficient. Treatment of $B R A F$-mutant CRC with single-agent vemurafenib cannot be recommended at this time.

In preclinical studies, mirroring the clinical findings, single-agent vemurafenib treatment of $B R A F$-mutant CRC cell lines yielded only transient inhibition of BRAF due to compensatory feedback activation of EGFR and its downstream pathways such as MEK and ERK. ${ }^{50,51}$ However, combination treatment of these $B R A F$-mutant CRC cell lines with both vemurafenib and EGFR-targeted drugs such as cetuximab or gefitinib (an EGFR tyrosine kinase inhibitor) resulted in more sustained inhibition of EGFR, BRAF, and its downstream pathways..$^{50,51}$

\section{Combination strategies targeting $B R A F$ in CRC}

On the basis of these laboratory findings, multiple clinical trials have been undertaken, which have demonstrated somewhat more promising evidence of activity after combination therapy targeting BRAF. Yaeger et $\mathrm{al}^{27}$ recently published findings from a pilot trial in which 15 patients with $B R A F$-mutant CRCs were treated with a combination of BRAF and EGFR inhibition. Of 12 evaluable patients, disease shrinkage was noted in ten patients, including two with a partial response. These responses, however, have been transient, with median progression-free survival of 3.2 months (95\% CI: 1.6-5.3 months). ${ }^{27}$ Preliminary findings from another study and a case report evaluating cetuximab and vemurafenib demonstrated similar results. ${ }^{52,53}$

Preliminary findings from other studies have also noted modest activity with combination therapies targeting EGFR, BRAF, and MEK. Specifically in a Phase I/II trial ${ }^{54}$ of the BRAF inhibitor dabrafenib and the MEK inhibitor trametinib, 43 patients with $B R A F$-mutant CRCs were treated with the combination and $27 / 43$ had disease control with stable disease $(n=22)$, partial response $(n=4)$, or complete response $(n=1)$.

In another study, ten patients with $B R A F$-mutant CRCs received combination therapy with dabrafenib, panitumumab, and trametinib targeting MEK. ${ }^{55}$ Of six evaluable patients receiving this triplet combination, four had partial responses and two had stable disease. Of nine patients with $B R A F$-mutant CRCs who received combination therapy with dabrafenib and panitumumab, 7/8 evaluable patients had stable disease. These combinations were reasonably well tolerated, with no Grade 4 or 5 events in patients receiving doublet therapy and one event each of $>$ Grade 3 vomiting, rash, and skin fissures, thought at least possibly related to drug. ${ }^{55}$ While preliminary results from the aforementioned studies are perhaps more promising than were seen with single-agent BRAF inhibitors, these responses are short lived for most patients.

\section{Mechanisms of resistance to BRAF inhibition in CRCs}

Given the transient responses to BRAF-targeted therapies in patients with CRCs, postprogression biopsies have been useful in identifying mechanisms of acquired resistance to BRAF-targeted therapies. Ahronian et al performed wholeexome sequencing on paired pretreatment and posttreatment samples from patients who underwent combination therapy directed at $B R A F$ alterations. ${ }^{56}$ In two patients with $B R A F$ mutant CRCs who received RAF inhibitors and EGFR inhibitors, posttreatment biopsies revealed the emergence of a $K R A S$ amplification in a first paired sample and a $B R A F$ amplification in a second sample. In a third paired sample from a patient with $B R A F$-mutant CRC treated with a RAF inhibitor and a MEK inhibitor, posttreatment biopsies demonstrated the development of mutations in both $M E K 1$ (F53L) and $A R A F$ (Q489L). In a tumor cell line harboring a $M E K 1$ F53L mutation and also in a tumor cell line derived from this patient's tumor, treatment with an ERK inhibitor both alone and in combination with a BRAF inhibitor could rescue the cells from resistance to the RAF and MEK inhibitors. Although limited numbers of samples have been tested here, these findings suggest a diverse pattern of acquired resistance mechanisms in patients with $B R A F$-mutant CRCs treated with agents targeting BRAF, EGFR, and MEK. These alterations, however, all remain centered on the RAS-RAF-MEK-ERK pathway, suggesting that continued inhibition of this pathway through alternate means may be a way to overcome this resistance. ${ }^{56}$ These findings also highlight the critical importance of obtaining paired samples and biopsies at the time of resistance on clinical trials of targeted agents.

\section{Future directions}

While mutated BRAF has been a powerful target in certain cancers, revolutionizing the way we treat selected patients with melanomas and lung cancers through single-agent use of RAF inhibitors in patients with tumors harboring alterations in $B R A F$, in $\mathrm{CRC}$, the treatment paradigm of using single-agent targeted therapy in a molecularly selected 
population has been thus far unsuccessful. The biological underpinnings of $B R A F$-mutant $\mathrm{CRC}$ appear more complex. Combination strategies targeting RAF, EGFR, and MEK have been more successful, with improved response rates, although the beneficial effects have been short lived. To better understand the mechanisms of acquired resistance in this population, whole-exome sequencing in paired pre- and posttreatment biopsies has been illustrative, demonstrating molecular alterations in the same pathway and suggesting that combinations and targeting of downstream kinases may rescue tumor cells from resistance.

While much progress has been made in our understanding of this molecular-defined subset of CRC, many unanswered questions remain. First, clinical trials in this population of patients have focused exclusively on the management of patients in the metastatic setting. Can we personalize the adjuvant treatment of patients with early-stage $B R A F$-mutant $\mathrm{CRC}$ to ultimately improve outcomes in a setting when this disease remains curable? Developing better treatment strategies to manage these patients in the early-stage setting is necessary, given that these patients' outcomes remain poor when they develop metastatic disease.

Second, BRAF-mutant CRCs have an association with mismatch repair-deficient CRCs, and recent data suggest that patients with mismatch repair-deficient $\mathrm{CRC}$ have high response rates, with durable responses to immunotherapies. ${ }^{57}$ Can patients with $B R A F$-mutant, mismatch repair-deficient $\mathrm{CRC}$ benefit from immunotherapy in this same way? Finally, we have seen promising but short-lived response in patients with $B R A F$-mutant CRC to targeted therapies. Developing tolerable combination treatment strategies with sustained inhibition against $B R A F$ and its associated pathways will be critical to meaningfully improve the outcomes for patients with this disease. Given the poor overall survival and outcomes for patients with this disease, continued research and efforts into treating this subgroup of patients with CRCs are warranted.

\section{Disclosure}

The authors report no conflicts of interest in this work.

\section{References}

1. Tol J, Nagtegaal ID, Punt CJ. BRAF mutation in metastatic colorectal cancer. N Engl J Med. 2009;361(1):98-99.

2. De Roock W, De Vriendt V, Normanno N, Ciardiello F, Tejpar S. KRAS, BRAF, PIK3CA, and PTEN mutations: implications for targeted therapies in metastatic colorectal cancer. Lancet Oncol. 2011;12(6):594-603.

3. Rizzo S, Bronte G, Fanale D, et al. Prognostic vs predictive molecular biomarkers in colorectal cancer: is KRAS and BRAF wild type status required for anti-EGFR therapy? Cancer Treat Rev. 2010;36(Suppl 3): S56-S61.
4. Siegel RL, Miller KD, Jemal A. Cancer statistics, 2015. CA Cancer J Clin. 2015;65(1):5-29.

5. Cantwell-Dorris ER, O'Leary JJ, Sheils OM. BRAFV600E: implications for carcinogenesis and molecular therapy. Mol Cancer Ther. 2011;10(3):385-394.

6. Pratilas CA, Xing F, Solit DB. Targeting oncogenic BRAF in human cancer. Curr Top Microbiol Immunol. 2012;355:83-98.

7. Vakiani E, Solit DB. KRAS and BRAF: drug targets and predictive biomarkers. J Pathol. 2011;223(2):219-229.

8. De Roock W, Claes B, Bernasconi D, et al. Effects of KRAS, BRAF, NRAS, and PIK3CA mutations on the efficacy of cetuximab plus chemotherapy in chemotherapy-refractory metastatic colorectal cancer: a retrospective consortium analysis. Lancet Oncol. 2010;11(8):753-762.

9. Irahara N, Baba Y, Nosho K, et al. NRAS mutations are rare in colorectal cancer. Diagn Mol Pathol. 2010;19(3):157-163.

10. Douillard JY, Oliner KS, Siena S, et al. Panitumumab-FOLFOX4 treatment and RAS mutations in colorectal cancer. $N$ Engl $J$ Med. 2013;369(11):1023-1034

11. Lenz HJ, Van Cutsem E, Khambata-Ford S, et al. Multicenter phase II and translational study of cetuximab in metastatic colorectal carcinoma refractory to irinotecan, oxaliplatin, and fluoropyrimidines. $J$ Clin Oncol. 2006;24(30):4914-4921.

12. Amado RG, Wolf M, Peeters M, et al. Wild-type KRAS is required for panitumumab efficacy in patients with metastatic colorectal cancer. $J$ Clin Oncol. 2008;26(10):1626-1634.

13. Van Cutsem E, Köhne CH, Hitre E, et al. Cetuximab and chemotherapy as initial treatment for metastatic colorectal cancer. N Engl J Med. 2009; 360(14):1408-1417.

14. Berger MF, Hodis E, Heffernan TP, et al. Melanoma genome sequencing reveals frequent PREX2 mutations. Nature. 2012;485(7399):502-506.

15. Kris MG, Johnson BE, Berry LD, et al. Using multiplexed assays of oncogenic drivers in lung cancers to select targeted drugs. JAMA. 2014; 311(19): 1998-2006.

16. Sosman JA, Kim KB, Schuchter L, et al. Survival in BRAF V600-mutant advanced melanoma treated with vemurafenib. $N$ Engl J Med. 2012; 366(8):707-714.

17. Chapman PB, Hauschild A, Robert C, et al. Improved survival with vemurafenib in melanoma with BRAF V600E mutation. $N$ Engl J Med. 2011;364(26):2507-2516

18. McArthur GA, Chapman PB, Robert C, et al. Safety and efficacy of vemurafenib in BRAF(V600E) and BRAF(V600K) mutation-positive melanoma (BRIM-3): extended follow-up of a phase 3, randomised, open-label study. Lancet Oncol. 2014;15(3):323-332.

19. Gautschi O, Pauli C, Strobel K, et al. A patient with BRAF V600E lung adenocarcinoma responding to vemurafenib. $J$ Thorac Oncol. 2012;7(10):e23-e24.

20. Peters S, Michielin O, Zimmermann S. Dramatic response induced by vemurafenib in a BRAF V600E-mutated lung adenocarcinoma. $J$ Clin Oncol. 2013;31(20):e341-e344.

21. Robinson SD, O'Shaughnessy JA, Cowey CL, Konduri K. BRAF V600Emutated lung adenocarcinoma with metastases to the brain responding to treatment with vemurafenib. Lung Cancer. 2014;85(2):326-330.

22. Planchard D, Kim TM, Mazieres J, et al. Dabrafenib in patients with BRAF V600E-mutant advanced non-small cell lung cancer (NSCLC): a multicenter, open-label, phase II trial (BRF113928). Ann Oncol. 2014;25(5): 1 .

23. Gonsalves WI, Mahoney MR, Sargent DJ, et al; Alliance for Clinical Trials in Oncology. Patient and tumor characteristics and BRAF and KRAS mutations in colon cancer, NCCTG/Alliance N0147. $J$ Natl Cancer Inst. 2014;106:7.

24. Yokota T, Ura T, Shibata N, et al. BRAF mutation is a powerful prognostic factor in advanced and recurrent colorectal cancer. Br J Cancer. 2011;104(5):856-862.

25. Van Cutsem E, Köhne CH, Láng I, et al. Cetuximab plus irinotecan, fluorouracil, and leucovorin as first-line treatment for metastatic colorectal cancer: updated analysis of overall survival according to tumor KRAS and BRAF mutation status. J Clin Oncol. 2011;29(15):2011-2019. 
26. Yaeger R, Cercek A, Chou JF, et al. BRAF mutation predicts for poor outcomes after metastasectomy in patients with metastatic colorectal cancer. Cancer. 2014;120(15):2316-2324.

27. Yaeger R, Cercek A, O'Reilly EM, et al. Pilot trial of combined BRAF and EGFR inhibition in BRAF-mutant metastatic colorectal cancer patients. Clin Can Res. 2015;21(6):1313-1320.

28. Lipsyc MD, Yaeger R, Dengel LT, Saltz L. Axillary lymph node involvement, a unique pattern of metastasis in BRAF-mutant colorectal cancer JAMA Oncol. Epub 2015 Apr 23.

29. Roth AD, Tejpar S, Delorenzi M, et al. Prognostic role of KRAS and BRAF in stage II and III resected colon cancer: results of the translational study on the PETACC-3, EORTC 40993, SAKK 60-00 trial. J Clin Oncol. 2010;28(3):466-474.

30. Souglakos J, Philips J, Wang R, et al. Prognostic and predictive value of common mutations for treatment response and survival in patients with metastatic colorectal cancer. Br J Cancer. 2009;101(3):465-472.

31. Popovici V, Budinska E, Tejpar S, et al. Identification of a poorprognosis BRAF-mutant-like population of patients with colon cancer. J Clin Oncol. 2012;30(12):1288-1295.

32. Richman SD, Seymour MT, Chambers P, et al. KRAS and BRAF mutations in advanced colorectal cancer are associated with poor prognosis but do not preclude benefit from oxaliplatin or irinotecan: results from the MRC FOCUS trial. J Clin Oncol. 2009;27(35):5931-5937.

33. Khambata-Ford S, Garrett CR, Meropol NJ, et al. Expression of epiregulin and amphiregulin and K-ras mutation status predict disease control in metastatic colorectal cancer patients treated with cetuximab. J Clin Oncol. 2007;25(22):3230-3237.

34. Yokota T. Are KRAS/BRAF mutations potent prognostic and/or predictive biomarkers in colorectal cancers? Anticancer Agents Med Chem 2012;12(2):163-171.

35. Bokemeyer C, Van Cutsem E, Rougier P, et al. Addition of cetuximab to chemotherapy as first-line treatment for KRAS wild-type metastatic colorectal cancer: pooled analysis of the CRYSTAL and OPUS randomised clinical trials. Eur J Cancer. 2012;48(10):1466-1475.

36. Di Nicolantonio F, Martini M, Molinari F, et al. Wild-type BRAF is required for response to panitumumab or cetuximab in metastatic colorectal cancer. J Clin Oncol. 2008;26(35):5705-5712.

37. Loupakis F, Ruzzo A, Cremolini C, et al. KRAS codon 61, 146 and BRAF mutations predict resistance to cetuximab plus irinotecan in KRAS codon 12 and 13 wild-type metastatic colorectal cancer. Br J Cancer. 2009;101(4):715-721.

38. Mao C, Liao RY, Qiu LX, Wang XW, Ding H, Chen Q. BRAF V600E mutation and resistance to anti-EGFR monoclonal antibodies in patients with metastatic colorectal cancer: a meta-analysis. Mol Biol Rep. 2011; 38(4):2219-2223.

39. Pietrantonio F, Petrelli F, Coinu A, et al. Predictive role of BRAF mutations in patients with advanced colorectal cancer receiving cetuximab and panitumumab: a meta-analysis. Eur J Cancer. 2015;51(5): 587-594.

40. National Comprehensive Cancer Network. NCCN Guidelines: Colon Cancer. Volume 2. Version 3. 2015. Available from :http://www.nccn. org/professionals/physician_gls/recently_updated.asp. Accessed August $12,2015$.

41. Varghese AM, Berger MF. Advancing clinical oncology through genome biology and technology. Genome Biol. 2014;15(8):427.

42. Dias-Santagata D, Akhavanfard S, David SS, et al. Rapid targeted mutational analysis of human tumours: a clinical platform to guide personalized cancer medicine. EMBO Mol Med. 2010;2(5):146-158.

Advances in Genomics and Genetics

\section{Publish your work in this journal}

Advances in Genomics and Genetics is an international, peer reviewed, open access journal that focuses on new developments in characterizing the human and animal genome and specific gene expressions in health and disease. Particular emphasis will be given to those studies that elucidate genes, biomarkers and targets in the development of new or improved therapeutic
43. MacConaill LE, Campbell CD, Kehoe SM, et al. Profiling critical cancer gene mutations in clinical tumor samples. PLoS One. 2009;4(11): e7887.

44. Thomas RK, Baker AC, Debiasi RM, et al. High-throughput oncogene mutation profiling in human cancer. Nat Genet. 2007;39(3): 347-351.

45. Cheng DT, Mitchell TN, Zehir A, et al. Memorial Sloan Ketteringintegrated mutation profiling of actionable cancer targets (MSKIMPACT): a hybridization capture-based next-generation sequencing clinical assay for solid tumor molecular oncology. J Mol Diagn. 2015; 17(3):251-264.

46. Frampton GM, Fichtenholtz A, Otto GA, et al. Development and validation of a clinical cancer genomic profiling test based on massively parallel DNA sequencing. Nat Biotechnol. 2013;31(11):1023-1031.

47. Wagle N, Berger MF, Davis MJ, et al. High-throughput detection of actionable genomic alterations in clinical tumor samples by targeted, massively parallel sequencing. Cancer Discov. 2012;2(1):82-93.

48. Renaud S, Romain B, Falcoz PE, et al. KRAS and BRAF mutations are prognostic biomarkers in patients undergoing lung metastasectomy of colorectal cancer. Br J Cancer. 2015;112(4):720-728.

49. Kopetz S, Desai J, Chan E, et al. PLX4032 in metastatic colorectal cancer patients with mutant BRAF tumors. J Clin Oncol. 2010;28(15s): sul;abstr3534.

50. Prahallad A, Sun C, Huang S, et al. Unresponsiveness of colon cancer to BRAF(V600E) inhibition through feedback activation of EGFR. Nature. 2012;483(7387):100-103.

51. Corcoran RB, Ebi H, Turke AB, et al. EGFR-mediated re-activation of MAPK signaling contributes to insensitivity of BRAF mutant colorectal cancers to RAF inhibition with vemurafenib. Cancer Discov. 2012;2(3):227-235.

52. Tabernero J, Chan E, Baselga J, et al. VE-BASKET, a Simon 2-stage adaptive design, phase II, histology-independent study in nonmelanoma solid tumors harboring BRAF V600 mutations: activity of vemurafenib with or without cetuximab in colorectal cancer. J Clin Oncol. 2014;32(5s):sul;abstr3518.

53. Connolly K, Brungs D, Szeto E, Epstein RJ. Anticancer activity of combination targeted therapy using cetuximab plus vemurafenib for refractory BRAF (V600E)-mutant metastatic colorectal carcinoma. Curr Oncol. 2014;21(1):e151-e154.

54. Corcoran RB, Atreya CE, Falchook GS, et al. Phase 1-2 trial of the BRAF inhibitor dabrafenib pluse MEK inhibitor trametinib in BRAF V600 mutant colorectal cancer: updated efficacy and biomarker analysis. J Clin Oncol. 2014;32(5s):sul;abstr3517.

55. Bendell JC, Atreya CE, Andre T, et al. Efficacy and tolerability in an open-label phsae I/II study of MEK inhibitor trametinib, BRAF inhibitor dabrafenib, and anti-EGFR antibody panitumumab in combination in patients with BRAF V600E mutated colorectal cancer. J Clin Oncol. 2014;32(5s):sul;abstr3515.

56. Ahronian LG, Sennott EM, Van Allen EM, et al. Clinical acquired resistance to RAF inhibitor combinations in BRAF-mutant colorectal cancer through MAPK pathway alterations. Cancer Discov. 2015;5(4): 358-367.

57. Le DT, Uram JN, Wang H, et al. PD-1 blockade in tumors with mismatch-repair deficiency. $N$ Engl J Med. 2015;372(26):2509-2520.

interventions. The journal is characterized by the rapid reporting of reviews, original research, methodologies, technologies and analytics in this subject area. The manuscript management system is completely online and includes a very quick and fair peer-review system. Visit http://www.dovepress.com/ testimonials.php to read real quotes from published authors. 